\title{
Prevalence of incidental lesions in pituitary, adrenals, pancreas and ovaries in a Sri Lankan cohort: Preliminary results of a post-mortem study
}

P. Katulanda ${ }^{1}$, A. A. H. Priyani ${ }^{2}$, M. V. C. de Silva ${ }^{2}$, A. Tennakoon ${ }^{3}$, N. Somasundaram ${ }^{4}$, R. R. G. S. Amararathna ${ }^{3}$, U. Attygalle ${ }^{3}$, H. D. Wijesinghe ${ }^{2}$, G. G, Ranaweera ${ }^{2}$, B. A. V. S. Bamunuarachchi $^{1}$, R. D. B. G. Madushanka ${ }^{1}$ and S. S. Madawanarachchi ${ }^{1}$

Diabetes Research Unit, Department of Clinical Medicine, University of Colombo, Sri Lanka ${ }^{1}$ Department of Pathology, Faculty of medicine, University of Colombo, Sri Lanka ${ }^{2}$ Institute of forensic Medicine and Toxicology, Colombo, Sri Lanka ${ }^{3}$ National Hospital of Sri Lanka ${ }^{4}$

DOI: http://doi.org/10.4038/jdp.v11i2.7722

\section{Introduction}

Prevalence of incidental pathological lesions in pituitary, adrenals, pancreas and ovaries is not well known in the Sri Lankan setting. This study aims to determine the prevalence of incidental pathological lesions in these organs.

\section{Methodology}

A prospective study of consecutive autopsies of males and females who died of undiagnosed acute diseases and accidents during the 2014-2016 period was done after obtaining informed written consent from next of kin. Deaths due to endocrine related diseases except diabetes mellitus and putrefied bodies were excluded. A brief history was obtained using an interviewer administered questionnaire. The entire pituitary, three sections each from head, body and tail of the pancreas, one section each from adrenals and ovaries and at least one section from all grossly abnormal lesions in the studied organs were examined histologically by a histopathologist.

\section{Results}

Two hundred and forty eight autopsies were studied including $174(70.16 \%)$ males and 74(29.83\%) females. Pituitary micro-adenoma and fibrosis with calcification were present in $2(0.8 \%)$ and $1(0.4 \%)$ cases respectively.
Increase in fat infiltration in the pancreas was present in $35(14.9 \%)$ cases and fibrosis in $12(5.1 \%)$. Four $(1.6 \%)$ had adrenal nodular hyperplasia and $2(0.8 \%)$ had granulomatous inflammation with caseous necrosis with no previous clinical history of tuberculosis. Of the autopsies of the 74 deceased females, $16(21.6 \%)$ had multiple cystic follicles and $2(2.7 \%)$ had haemorrhagic cysts.

\section{Conclusion}

The prevalence of incidental tumours in pituitary, adrenals, pancreas and ovaries were very low. However, non neoplastic pathological conditions were more commonly encountered. 